TIFR/TH/02-15

$\mathrm{PM} / 02-15$

\title{
Bell inequalities in four dimensional phase space and the three marginal theorem*.
}

\author{
G. Auberson ${ }^{\dagger}$ \\ Laboratoire de Physique Mathématique, UMR 5825-CNRS, \\ Université Montpellier II, \\ F-34095 Montpellier, Cedex 05, FRANCE. \\ G. Mahoux \\ Service de Physique Théorique, \\ Centre d'Études Nucléaires de Saclay, \\ F-91191 Gif-sur-Yvette Cedex, FRANCE. \\ S.M. Roy ${ }^{\S}$ and Virendra Singh \\ Department of Theoretical Physics, \\ Tata Institute of Fundamental Research, \\ Homi Bhabha Road, Mumbai 400 005, INDIA.
}

May 28, 2002.

*Work supported by the Indo-French Centre for the Promotion of Advanced Research, Project Nb 1501-02.

${ }^{\dagger}$ e-mail: auberson@lpm.univ-monpt2.fr

${ }_{\ddagger} \mathrm{e}$-mail: mahoux@spht.saclay.cea.fr

$\S$ e-mail: shasanka@theory.tifr.res.in

๑e-mail: vsingh@theory.tifr.res.in 


\begin{abstract}
We address the classical and quantum marginal problems, namely the question of simultaneous realizability through a common probability density in phase space of a given set of compatible probability distributions. We consider only distributions authorized by quantum mechanics, i.e. those corresponding to complete commuting sets of observables. For four-dimensional phase space with position variables $\vec{q}$ and momentum variables $\vec{p}$, we establish the two following points: i) given four compatible probabilities for $\left(q_{1}, q_{2}\right),\left(q_{1}, p_{2}\right),\left(p_{1}, q_{2}\right)$ and $\left(p_{1}, p_{2}\right)$, there does not always exist a positive phase space density $\rho(\vec{q}, \vec{p})$ reproducing them as marginals; this settles a long standing conjecture; it is achieved by first deriving Bell-like inequalities in phase space which have their own theoretical and experimental interest. ii) given instead at most three compatible probabilities, there always exist an associated phase space density $\rho(\vec{q}, \vec{p})$; the solution is not unique and its general form is worked out. These two points constitute our "three marginal theorem".
\end{abstract}

PACS : 03.65.Ta, 03.67.-a 


\section{Introduction}

In classical mechanics position and momentum can be simultaneously specified. Hence phase space density has a well defined meaning in classical statistical mechanics. In quantum theory the probability density for observing eigenvalues of a complete commuting set (CCS) of observables is specific to the experimental context for measuring that CCS. Joint probabilities for different CCS which contain mutually noncommuting operators are not defined. For example for a 2-dimensional configuration space, with $\vec{q}, \vec{p}$ denoting position and momentum, probability densities of anyone of the four CCS $\left(q_{1}, q_{2}\right),\left(q_{1}, p_{2}\right),\left(p_{1}, q_{2}\right)$ or $\left(p_{1}, p_{2}\right)$ are defined, but not their joint probabilities. The question one may raise is : can one define such joint probabilities, e.g. a phase space probability density $\rho(\vec{q}, \vec{p})$ such that all its marginals $\rrbracket^{-}$coincide with the quantum mechanical probabilities for the different individual CCS? This general question was first raised by Martin and Roy [1].

The Martin-Roy contextuality theorem demonstrates the impossibility of realizing quantum probability densities of all possible choices of the CCS of observables as marginals of one positive definite phase space density. For example, consider a two dimensional configuration space. Let coordinates $q_{1 \alpha}, q_{2 \alpha}$ be obtained from $q_{1}, q_{2}$ by a rotation of arbitrary angle $\alpha$, and momenta $p_{1 \alpha}, p_{2 \alpha}$ be related similarly to $p_{1}, p_{2}$

$$
\left(\begin{array}{c}
q_{1 \alpha} \\
q_{2 \alpha}
\end{array}\right)=V\left(\begin{array}{c}
q_{1} \\
q_{2}
\end{array}\right),\left(\begin{array}{c}
p_{1 \alpha} \\
p_{2 \alpha}
\end{array}\right)=V\left(\begin{array}{c}
p_{1} \\
p_{2}
\end{array}\right)
$$

where

$$
V=\left(\begin{array}{cc}
\cos \alpha & \sin \alpha \\
-\sin \alpha & \cos \alpha
\end{array}\right)
$$

Does there exist for every quantum state (with density operator $\widehat{\rho}$ ) a positive definite phase space density $\rho(\vec{q}, \vec{p})$ such that its marginals agree with the corresponding quantum probabilities, i.e.,

$$
\int d p_{1 \alpha} d q_{2 \alpha} \rho(\vec{q}, \vec{p})=\left\langle q_{1 \alpha}, p_{2 \alpha}|\widehat{\rho}| q_{1 \alpha}, p_{2 \alpha}\right\rangle
$$

for all $\alpha$ ranging from 0 to $2 \pi$ ? They answered this question in the negative by finding a state $\hat{\rho}$ for which eqs. (I.3) for all $\alpha$ are inconsistent with positivity of $\rho$. Since different $\alpha$ correspond to different experimental contexts, the Martin-Roy theorem is a new Gleason-Kochen-Specker type contextuality theorem [2]. The positivity of the phase space density $\rho(\vec{q}, \vec{p})$ is absolutely crucial for this theorem; otherwise the Wigner distribution function [3] would be a solution of ([.3).

Equations (I.3) constitute conditions on an infinite set of marginals of $\rho(\vec{q}, \vec{p})$ (corresponding to the continuously infinite choices for $\alpha$ ) to agree with corresponding quantum probability densities. Their inconsistency still leaves open the question of consistency of a finite number of such marginal conditions.

\footnotetext{
${ }^{1}$ In agreement with common terminology, by "marginal" of a distribution over several variables, we denote integrals of the distribution over a subset of its variables.
} 
Indeed, the consistency of two marginal conditions where the marginals involve only nonintersecting sets of variables has been known for some time. Cohen and Zaparovanny [4] constructed the most general positive $\rho(\vec{q}, \vec{p})$ obeying

$$
\int d \vec{p} \rho(\vec{q}, \vec{p})=\langle\vec{q}|\hat{\rho}| \vec{q}\rangle, \quad \int d \vec{q} \rho(\vec{q}, \vec{p})=\langle\vec{p}|\widehat{\rho}| \vec{p}\rangle
$$

Their solutions generalize the obvious simple uncorrelated solution for pure states $\psi$,

$$
\rho(\vec{q}, \vec{p})=|\psi(\vec{q})|^{2}|\tilde{\psi}(\vec{p})|^{2},
$$

where tilda denotes Fourier transform. Based on generalized phase space densities exhibiting position momentum correlations, Roy and Singh [5] constructed a causal quantum mechanics reproducing quantum position and momentum probability densities, thus improving on De Broglie-Bohm mechanics [6] which only reproduced the quantum position probability densities. Later, going much further than the nonintersecting marginals of Cohen et al. [4], Roy and Singh [7] constructed a causal quantum mechanics based on a positive $\rho(\vec{q}, \vec{p})$ whose marginals reproduce the quantum probability densities of a chain of $N+1$ different CCS, e.g.

$$
\left(Q_{1}, Q_{2}, \cdots, Q_{N}\right),\left(P_{1}, Q_{2}, \cdots, Q_{N}\right),\left(P_{1}, P_{2}, Q_{3}, \cdots, Q_{N}\right), \cdots\left(P_{1}, P_{2}, \cdots, P_{N}\right) .
$$

Here $N$ is the dimension of the configuration space, and each CCS in the chain is obtained from the preceding one by replacing one of the position operators $Q_{i}$ by the conjugate momentum operator $P_{i}$.

Roy and Singh proposed the following definition: a Maximally Realistic Causal Quantum Mechanics is a causal mechanics which simultaneously reproduces the quantum probability densities of the maximum number of different (mutually noncommuting) CCS of observables as marginals of the same positive definite phase space density. They also conjectured that for $N$ dimensional configuration space this maximum number is $N+1$.

A proof of this long standing conjecture is important for quantum mechanics where it quantifies the extent of simultaneous realizability of non commuting CCS.

In this paper, we restrict ourselves to the case of $N=2$ degrees of freedom. The general case $(N>2)$ will be dealt with in a forthcoming paper. In Section II below, we first state the classical and quantum marginal problems and second, show that, given four classical compatible two-variable probability distributions, there does not always exist a positive phase space distribution reproducing them as marginals. In Section III, we develop a new tool, "the phase space Bell inequalities", which are the phase space analogues of the standard Bell inequalities [8] for a system of two spinhalf particles. We use them in Section IV to prove the conjecture for four-dimensional phase space $(N=2)$, namely the impossibility of simultaneous realization of quantum probabilities of more than three CCS as marginals. In Section V, we explicitly construct the most general phase space distribution which reproduces probabilities of three CSS as marginals. These results, the three marginal theorem, are relevant for the construction of maximally realistic quantum mechanics.

As our results are essentially new theorems for multidimensional Fourier transforms, they are also expected to be useful for classical signal and image processing [9]. 
The theorems of the present paper and their generalizations to arbitrary $N$ [10] considerably advance previous results in the field, which have only dealt with nonintersecting sets of marginals (e.g. time and frequency). A summary of the results of this paper without detailed proofs is being reported separately [11].

\section{Four marginal problem}

Let us consider a physical system with 2-dimensional configuration space. Let $\left(q_{1}, p_{1}\right)$ and $\left(q_{2}, p_{2}\right)$ be a set of canonical variables in the corresponding phase space. We look for a (normalized) probability distribution $\rho\left(q_{1}, q_{2}, p_{1}, p_{2}\right)$ such that

$$
\begin{aligned}
\rho\left(q_{1}, q_{2}, p_{1}, p_{2}\right) & \geq 0 \\
\int d p_{1} d p_{2} \rho\left(q_{1}, q_{2}, p_{1}, p_{2}\right) & =R\left(q_{1}, q_{2}\right), \\
\int d p_{1} d q_{2} \rho\left(q_{1}, q_{2}, p_{1}, p_{2}\right) & =S\left(q_{1}, p_{2}\right), \\
\int d q_{1} d p_{2} \rho\left(q_{1}, q_{2}, p_{1}, p_{2}\right) & =T\left(p_{1}, q_{2}\right), \\
\int d q_{1} d q_{2} \rho\left(q_{1}, q_{2}, p_{1}, p_{2}\right) & =U\left(p_{1}, p_{2}\right),
\end{aligned}
$$

where the four marginals $R\left(q_{1}, q_{2}\right), S\left(q_{1}, p_{2}\right), T\left(p_{1}, q_{2}\right)$, and $U\left(p_{1}, p_{2}\right)$, the respective joint probabilities, are given. For consistency we must have

$$
R, S, T, U \geq 0
$$

and

$$
\begin{aligned}
\int d q_{2} R\left(q_{1}, q_{2}\right) & =\int d p_{2} S\left(q_{1}, p_{2}\right), \\
\int d q_{1} R\left(q_{1}, q_{2}\right) & =\int d p_{1} T\left(p_{1}, q_{2}\right), \\
\int d q_{1} S\left(q_{1}, p_{2}\right) & =\int d p_{1} U\left(p_{1}, p_{2}\right), \\
\int d q_{2} T\left(p_{1}, q_{2}\right) & =\int d p_{2} U\left(p_{1}, p_{2}\right) .
\end{aligned}
$$

We shall refer to the problem Given four distributions $R, S, T$ and $U$, satisfying the consistency conditions, does there always exist a positive $\rho\left(q_{1}, q_{2}, p_{1}, p_{2}\right)$ with these distributions as marginals? as the Classical four marginal problem.

When the system is quantum mechanical and is described by a state vector $|\Psi\rangle$, each of the four marginals involves a pair of compatible observables and we have

$$
\begin{aligned}
R\left(q_{1}, q_{2}\right) & =\left|\left\langle q_{1}, q_{2}|\widehat{\rho}| q_{1}, q_{2}\right\rangle\right|^{2}, \\
S\left(q_{1}, p_{2}\right) & =\left|\left\langle q_{1}, p_{2}|\widehat{\rho}| q_{1}, p_{2}\right\rangle\right|^{2}, \\
T\left(p_{1}, q_{2}\right) & =\left|\left\langle p_{1}, q_{2}|\widehat{\rho}| p_{1}, q_{2}\right\rangle\right|^{2}, \\
U\left(p_{1}, p_{2}\right) & =\left|\left\langle p_{1}, p_{2}|\widehat{\rho}| p_{1}, p_{2}\right\rangle\right|^{2} .
\end{aligned}
$$


In this case, the above consistency conditions are automatically satisfied. We then refer to the problem as the Quantum four marginal problem. A positive answer to it for all states $\hat{\rho}$ would mean that a realistic interpretation of the quantum results is possible (to the extent that only measurements connected to the four marginals are involved).

We shall see that the answer to both problems is negative.

Let us first show that the classical four marginal problem does not always admit a solution. To this end, consider the following set of marginals

$$
\begin{aligned}
R\left(q_{1}, q_{2}\right) & =\frac{1}{2}\left[\delta\left(q_{1}-a_{1}\right) \delta\left(q_{2}-a_{2}\right)+\delta\left(q_{1}-a_{1}^{\prime}\right) \delta\left(q_{2}-a_{2}^{\prime}\right)\right], \\
S\left(q_{1}, p_{2}\right) & =\frac{1}{2}\left[\delta\left(q_{1}-a_{1}\right) \delta\left(p_{2}-b_{2}\right)+\delta\left(q_{1}-a_{1}^{\prime}\right) \delta\left(p_{2}-b_{2}^{\prime}\right)\right] \\
T\left(p_{1}, q_{2}\right) & =\frac{1}{2}\left[\delta\left(p_{1}-b_{1}\right) \delta\left(q_{2}-a_{2}\right)+\delta\left(p_{1}-b_{1}^{\prime}\right) \delta\left(q_{2}-a_{2}^{\prime}\right)\right], \\
U\left(p_{1}, p_{2}\right) & =\frac{1}{2}\left[\delta\left(p_{1}-b_{1}\right) \delta\left(p_{2}-b_{2}^{\prime}\right)+\delta\left(p_{1}-b_{1}^{\prime}\right) \delta\left(p_{2}-b_{2}\right)\right]
\end{aligned}
$$

which obviously satisfy the consistency conditions ([I.6) and (II.7). They possess two essential features. First, their non factorized form. Second, in view of the expressions of $R, S$ and $T$, the positions of the factors $\delta\left(p_{2}-b_{2}\right)$ and $\delta\left(p_{2}-b_{2}^{\prime}\right)$ in the expression of $U$ are not the "natural ones".

Eq.(II.9) means that the support of the distribution $R$ in the plane $\left(q_{1}, q_{2}\right)$ consists in the two points $\left(a_{1}, a_{2}\right)$ and $\left(a_{1}^{\prime}, a_{2}^{\prime}\right)$. As a consequence, any positive $\rho$ satisfying (II.2) should have a support the projection of which on the plane $\left(q_{1}, q_{2}\right)$ would also consist in those two points. That is

$$
\rho=\delta\left(q_{1}-a_{1}\right) \delta\left(q_{2}-a_{2}\right) \alpha\left(p_{1}, p_{2}\right)+\delta\left(q_{1}-a_{1}^{\prime}\right) \delta\left(q_{2}-a_{2}^{\prime}\right) \alpha^{\prime}\left(p_{1}, p_{2}\right),
$$

where $\alpha$ and $\alpha^{\prime}$ are some positive distributions. Similarly, from eqs.([II.3) to ([I.5)

$$
\begin{aligned}
\rho & =\delta\left(q_{1}-a_{1}\right) \delta\left(p_{2}-b_{2}\right) \beta\left(p_{1}, q_{2}\right)+\delta\left(q_{1}-a_{1}^{\prime}\right) \delta\left(p_{2}-b_{2}^{\prime}\right) \beta^{\prime}\left(p_{1}, q_{2}\right) \\
& =\delta\left(p_{1}-b_{1}\right) \delta\left(q_{2}-a_{2}\right) \gamma\left(q_{1}, p_{2}\right)+\delta\left(p_{1}-b_{1}^{\prime}\right) \delta\left(q_{2}-a_{2}^{\prime}\right) \gamma^{\prime}\left(q_{1}, p_{2}\right) \\
& =\delta\left(p_{1}-b_{1}\right) \delta\left(p_{2}-b_{2}^{\prime}\right) \eta\left(q_{1}, q_{2}\right)+\delta\left(p_{1}-b_{1}^{\prime}\right) \delta\left(p_{2}-b_{2}\right) \eta^{\prime}\left(q_{1}, q_{2}\right)
\end{aligned}
$$

According to eqs. (II.13) to (II.15)

$$
\begin{aligned}
\rho= & v \delta\left(q_{1}-a_{1}\right) \delta\left(q_{2}-a_{2}\right) \delta\left(p_{1}-b_{1}\right) \delta\left(p_{2}-b_{2}\right) \\
& +v^{\prime} \delta\left(q_{1}-a_{1}^{\prime}\right) \delta\left(q_{2}-a_{2}^{\prime}\right) \delta\left(p_{1}-b_{1}^{\prime}\right) \delta\left(p_{2}-b_{2}^{\prime}\right),
\end{aligned}
$$

with $v \geq 0, v^{\prime} \geq 0,\left(v+v^{\prime}=1\right)$. Clearly, eqs.(II.16) and (II.17) are incompatible, which establishes the non existence of $\rho$, and settles the classical four marginal problem.

This however does not settle the quantum problem. Actually, the above example obviously cannot be strictly realized through a wave function in accordance with eqs.(II.8). More than that, this example is so "twisted" that, even after smoothing out the $\delta$ measures in eqs.(II.9) to (II.12), approaching it close enough through a wave function appears as very difficult (if not impossible). Instead, we develop a new mathematical tool. 


\section{Phase space Bell inequalities}

Consider any choice of functions $r\left(q_{1}, q_{2}\right), s\left(q_{1}, p_{2}\right), t\left(p_{1}, q_{2}\right)$, and $u\left(p_{1}, p_{2}\right)$, obeying

$$
A \leq r\left(q_{1}, q_{2}\right)+s\left(q_{1}, p_{2}\right)+t\left(p_{1}, q_{2}\right)+u\left(p_{1}, p_{2}\right) \leq B \quad\left(\forall q_{1}, q_{2}, p_{1}, p_{2}\right),
$$

Multiply by $\rho(\vec{q}, \vec{p})$, integrate over phase space and use positivity and normalization of $\rho(\vec{q}, \vec{p})$. We deduce that the (classical as well as quantum) four marginal problem cannot have a solution unless

$$
\begin{aligned}
A \leq & \int d q_{1} d q_{2} r\left(q_{1}, q_{2}\right) R\left(q_{1}, q_{2}\right)+\int d q_{1} d p_{2} s\left(q_{1}, p_{2}\right) S\left(q_{1}, p_{2}\right) \\
& +\int d p_{1} d q_{2} t\left(p_{1}, q_{2}\right) T\left(p_{1}, q_{2}\right)+\int d p_{1} d p_{2} u\left(p_{1}, p_{2}\right) U\left(p_{1}, p_{2}\right) \leq B .
\end{aligned}
$$

Here $R, S, T$ and $U$ are defined by eqs.(II.8) in the quantum case. It turns out that a particularly interesting choice is

$$
\begin{aligned}
r\left(q_{1}, q_{2}\right) & =\operatorname{sgn} F_{1}\left(q_{1}\right) \operatorname{sgn} F_{2}\left(q_{2}\right), \\
s\left(q_{1}, p_{2}\right) & =\operatorname{sgn} F_{1}\left(q_{1}\right) \operatorname{sgn} G_{2}\left(p_{2}\right), \\
t\left(p_{1}, q_{2}\right) & =\operatorname{sgn} G_{1}\left(p_{1}\right) \operatorname{sgn} F_{2}\left(q_{2}\right), \\
u\left(p_{1}, p_{2}\right) & =-\operatorname{sgn} G_{1}\left(p_{1}\right) \operatorname{sgn} G_{2}\left(p_{2}\right),
\end{aligned}
$$

with $A=-2, B=+2$ and with $F_{1}, F_{2}, G_{1}$ and $G_{2}$ arbitrary non vanishing functions? Then the inequalities (III.2) become a phase space analogue of the Bell inequalities for spin variables.

The necessary conditions (III.2) provide us with an alternative proof that the classical problem does not always admit a solution. Indeed, it is readily seen that they are violated for the marginals (II.9) to (II.12) and functions $F$ 's and G's such that

$$
\begin{aligned}
& F_{1}\left(a_{1}\right), F_{2}\left(a_{2}\right), G_{1}\left(b_{1}\right), G_{2}\left(b_{2}\right)>0, \\
& F_{1}\left(a_{1}^{\prime}\right), F_{2}\left(a_{2}^{\prime}\right), G_{1}\left(b_{1}^{\prime}\right), G_{2}\left(b_{2}^{\prime}\right)<0 .
\end{aligned}
$$

We shall see in the next section that the necessary conditions (III.2) can be violated also in the quantum case. There, the analogy between our correlation inequalities (III.2) (with the choice (III.3)) and Bell inequalities will become more apparent, especially as regards to their implications.

\section{Solving the four marginal quantum problem}

This section is divided into four parts. In the first one, we prove the existence of wave functions which violate the correlation inequalities (III.2). Strictly speaking, this already settles the problem. However, the explicit construction of such wave

\footnotetext{
${ }^{2}$ Note that, with this choice, the sum $r+s+t+u$ assumes only its two extremal values $A=-2$ and $B=+2$, which makes it in a sense optimal.
} 
functions, which we present in subsections B and C, is worthwhile in that it exhibits the physical implications of our inequalities. In subsection $\mathrm{D}$, we elaborate on the formal analogy with Bell inequalities.

\section{IV-A. Non constructive proof}

One first notices that $\chi_{1}\left(q_{1}\right) \equiv \frac{1}{2}\left[1+\operatorname{sgn} F_{1}\left(q_{1}\right)\right]$ is the characteristic function of some set $S_{1} \subset \mathbb{R}$, and similarly for $F_{2}, G_{1}$ and $G_{2}$, so that eqs.(III.3) read

$$
\begin{aligned}
r\left(q_{1}, q_{2}\right) & =\left(2 \chi_{1}-1\right)\left(2 \chi_{2}-1\right), \\
s\left(q_{1}, p_{2}\right) & =\left(2 \chi_{1}-1\right)\left(2 \chi_{2}^{\prime}-1\right), \\
t\left(p_{1}, q_{2}\right) & =\left(2 \chi_{1}^{\prime}-1\right)\left(2 \chi_{2}-1\right), \\
u\left(p_{1}, p_{2}\right) & =-\left(2 \chi_{1}^{\prime}-1\right)\left(2 \chi_{2}^{\prime}-1\right),
\end{aligned}
$$

where $\chi_{i}$ stands for $\chi_{i}\left(q_{i}\right)$ and $\chi_{i}^{\prime}$ for $\chi_{i}^{\prime}\left(p_{i}\right),(i=1,2)$. Inequalities (III.1) then become

$$
0 \leq \mathcal{P} \leq 1
$$

and in fact $\mathcal{P}(1-\mathcal{P})=0$ (see footnote 2), where $\mathcal{P}\left(q_{1}, q_{2}, p_{1}, p_{2}\right)$ is given by

$$
\mathcal{P}=\chi_{1}+\chi_{2}+\chi_{1}^{\prime} \chi_{2}^{\prime}-\chi_{1} \chi_{2}-\chi_{1} \chi_{2}^{\prime}-\chi_{1}^{\prime} \chi_{2} .
$$

Let us define a corresponding quantum operator $\widehat{\mathcal{P}}$ by

$$
\widehat{\mathcal{P}}=\widehat{\chi}_{1}+\widehat{\chi}_{2}+\widehat{\chi}_{1}^{\prime} \widehat{\chi}_{2}^{\prime}-\widehat{\chi}_{1} \widehat{\chi}_{2}-\widehat{\chi}_{1} \widehat{\chi}_{2}^{\prime}-\widehat{\chi}_{1}^{\prime} \widehat{\chi}_{2}
$$

where

$$
\begin{aligned}
& \widehat{\chi}_{1}=\int_{S_{1}} d q_{1}\left|q_{1}\right\rangle\left\langle q_{1}\right| \otimes \mathbf{1}_{2}, \\
& \widehat{\chi}_{2}=\mathbf{1}_{1} \otimes \int_{S_{2}} d q_{2}\left|q_{2}\right\rangle\left\langle q_{2}\right|, \\
& \widehat{\chi}_{1}^{\prime}=\int_{S_{1}^{\prime}} d p_{1}\left|p_{1}\right\rangle\left\langle p_{1}\right| \otimes \mathbf{1}_{2}, \\
& \widehat{\chi}_{2}^{\prime}=\mathbf{1}_{1} \otimes \int_{S_{2}^{\prime}} d p_{2}\left|p_{2}\right\rangle\left\langle p_{2}\right| .
\end{aligned}
$$

The $\widehat{\chi}$ 's are orthogonal projectors f $^{\prime}\left(\widehat{\chi} \dagger=\widehat{\chi}, \widehat{\chi}^{2}=\widehat{\chi}\right)$ acting on $\mathcal{H} \equiv L^{2}\left(\mathbb{R}, d q_{1}\right) \otimes$ $L^{2}\left(\mathbb{R}, d q_{2}\right)$. The product of two of them involving different indices commutes, so that $\widehat{\mathcal{P}}$ is a (bounded) self-adjoint operator.

The inequalities (III.2) to be tested in the quantum context then become, for pure states $\widehat{\rho}=|\Psi\rangle\langle\Psi|$,

$$
0 \leq\langle\Psi|\widehat{\mathcal{P}}| \Psi\rangle \leq 1 \quad \forall|\Psi\rangle \in \mathcal{H} \text { with }\langle\Psi \mid \Psi\rangle=1,
$$

\footnotetext{
${ }^{3}$ In eqs. IV.5), $S_{1}, S_{2}, S_{1}^{\prime}, S_{2}^{\prime}$ are the supports of $\widehat{\chi}_{1}, \widehat{\chi}_{2}, \widehat{\chi}_{1}^{\prime}, \widehat{\chi}_{2}^{\prime}$ respectively. Also, $\int_{S_{1}} d q_{1}\left|q_{1}\right\rangle\left\langle q_{1}\right|$ is, in standard Dirac notation, the orthogonal projection $\psi\left(q_{1}\right) \rightarrow \chi_{1}\left(q_{1}\right) \psi\left(q_{1}\right)$, whereas $\int_{S_{1}^{\prime}} d p_{1}\left|p_{1}\right\rangle\left\langle p_{1}\right|$ is the orthogonal projection $\tilde{\psi}\left(p_{1}\right) \rightarrow \chi_{1}^{\prime}\left(p_{1}\right) \tilde{\psi}\left(p_{1}\right), \tilde{\psi}\left(p_{1}\right)$ being the Fourier transform of $\psi\left(q_{1}\right)$, and so on.
} 
or, equivalently,

$$
\widehat{\mathcal{P}} \geq 0 \quad \text { and } \quad \mathbf{1}-\widehat{\mathcal{P}} \geq 0 \quad \text { in the operator sense. }
$$

Because $\widehat{\chi}_{j}$ fails to commute with $\widehat{\chi}_{j}^{\prime}(j=1,2), \widehat{\mathcal{P}}$ is not an orthogonal projector (see below), in contrast to the classical equality $\mathcal{P}^{2}=\mathcal{P}$. Exploiting this fact leads to the

Proposition 1 The operators $\widehat{\mathcal{P}}$ and $(\mathbf{1}-\widehat{\mathcal{P}})$ cannot be both positive.

As a consequence, there is at least one $|\Psi\rangle \neq 0$ such that the inequalities $\langle\Psi|\widehat{\mathcal{P}}| \Psi\rangle \geq 0$ and $\langle\Psi|(\mathbf{1}-\widehat{\mathcal{P}})| \Psi\rangle \geq 0$ cannot be simultaneously true. This just means that one of the two inequalities (IV.6) is violated for that $|\Psi\rangle$, which settles the question.

Proof of proposition 1:

Assume that $\widehat{\mathcal{P}}$ and $(\mathbf{1}-\widehat{\mathcal{P}})$ are both positive. This would imply

$$
\widehat{\mathcal{P}}(\mathbf{1}-\widehat{\mathcal{P}}) \geq 0,
$$

(remember that the product of two positive commuting operators is positive).

Now, a straightforward calculation of $\widehat{\mathcal{P}}^{2}$ from eq.(IV.4) yields

$$
\widehat{\mathcal{P}}^{2}=\widehat{\mathcal{P}}-\left[\widehat{\chi}_{1}, \widehat{\chi}_{1}^{\prime}\right]\left[\widehat{\chi}_{2}, \widehat{\chi}_{2}^{\prime}\right]
$$

and eq.(IV.8) would mean that $\left[\widehat{\chi}_{1}, \widehat{\chi}_{1}^{\prime}\right]\left[\widehat{\chi}_{2}, \widehat{\chi}_{2}^{\prime}\right]$ is a positive operator. That this is wrong is not surprising. Let us show it. Take a factorized $|\Psi\rangle$, namely $|\Psi\rangle=\left|\Phi_{1}\right\rangle \otimes$ $\left|\Phi_{2}\right\rangle$, so that

$$
\langle\Psi|\widehat{\mathcal{P}}(\mathbf{1}-\widehat{\mathcal{P}})| \Psi\rangle=-\left\langle\Phi_{1}\left|i\left[\widehat{\chi}_{1}, \widehat{\chi}_{1}^{\prime}\right]\right| \Phi_{1}\right\rangle\left\langle\Phi_{2}\left|i\left[\widehat{\chi}_{2}, \widehat{\chi}_{2}^{\prime}\right]\right| \Phi_{2}\right\rangle .
$$

It is enough to show that, for a given choice of the characteristic functions $\chi$ and $\chi^{\prime}$, the real number

$$
R[\Phi] \equiv\left\langle\Phi\left|i\left[\hat{\chi}, \hat{\chi}^{\prime}\right]\right| \Phi\right\rangle
$$

can assume both signs when $|\Phi\rangle$ is varied.

Let us define

$$
\left|\Phi^{+}\right\rangle=\widehat{\chi}|\Phi\rangle, \quad\left|\Phi^{-}\right\rangle=(\mathbf{1}-\widehat{\chi})|\Phi\rangle
$$

Using the identity

$$
\left[\widehat{\chi}, \widehat{\chi}^{\prime}\right]=\widehat{\chi} \widehat{\chi}^{\prime}(\mathbf{1}-\widehat{\chi})-(\mathbf{1}-\widehat{\chi}) \widehat{\chi}^{\prime} \widehat{\chi}
$$

gives $R[\Phi]$ the form

$$
R[\Phi]=i\left\langle\Phi^{+}\left|\widehat{\chi}^{\prime}\right| \Phi^{-}\right\rangle-i\left\langle\Phi^{-}\left|\widehat{\chi}^{\prime}\right| \Phi^{+}\right\rangle .
$$

Obviously, for $|\widetilde{\Phi}\rangle=\left|\Phi^{+}\right\rangle-\left|\Phi^{-}\right\rangle$, one has $R[\widetilde{\Phi}]=-R[\Phi]$.

This concludes the proof.

Remarks:

1) When the wave function $|\Psi\rangle$ factorizes, i.e. $\Psi\left(q_{1}, q_{2}\right)=\Phi_{1}\left(q_{1}\right) \Phi_{2}\left(q_{2}\right)$, a corresponding probability distribution $\rho$ always exists, namely

$$
\rho\left(q_{1}, q_{2}, p_{1}, p_{2}\right)=\left|\Phi_{1}\left(q_{1}\right)\right|^{2}\left|\Phi_{2}\left(q_{2}\right)\right|^{2}\left|\tilde{\Phi}_{1}\left(p_{1}\right)\right|^{2}\left|\tilde{\Phi}_{2}\left(p_{2}\right)\right|^{2},
$$


where the $\tilde{\Phi}_{i}$ 's are the Fourier transforms

$$
\tilde{\Phi}_{i}\left(p_{i}\right)=\frac{1}{\sqrt{2 \pi}} \int_{-\infty}^{+\infty} d q_{i} \mathrm{e}^{-i p_{i} q_{i}} \Phi_{i}\left(q_{i}\right), \quad(i=1,2) .
$$

Of course, this implies that eqs.(IV.6) are automatically satisfied for such factorized $|\Psi\rangle$ 's (which can also be checked from eq.([IV.4)).

2) The fact (used in the proof) that $\langle\Psi|\widehat{\mathcal{P}}(\mathbf{1}-\widehat{\mathcal{P}})| \Psi\rangle<0$ for some factorized $|\Psi\rangle$ 's is not inconsistent with the inequalities $0 \leq\langle\Psi|\widehat{\mathcal{P}}| \Psi\rangle \leq 1$ which are satisfied for those $|\Psi\rangle$ 's.

\section{IV-B. Construction}

We want to find wave functions $|\Psi\rangle$ violating the inequalities (IV.6). According to the first of the above remarks, one has to depart from the class of factorized $|\Psi\rangle$ 's. The simplest way to do it is to take just a sum of two such products.

Choose first

$$
S_{1}=S_{2} \equiv S \quad \text { and } \quad S_{1}^{\prime}=S_{2}^{\prime} \equiv S^{\prime}
$$

so that

$$
\widehat{\mathcal{P}}=\widehat{\chi} \otimes \mathbf{1}_{2}+\mathbf{1}_{1} \otimes \widehat{\chi}+\widehat{\chi}^{\prime} \otimes \widehat{\chi}^{\prime}-\widehat{\chi} \otimes \widehat{\chi}-\widehat{\chi} \otimes \widehat{\chi}^{\prime}-\widehat{\chi}^{\prime} \otimes \widehat{\chi}
$$

Take next

$$
\left\{\begin{array}{l}
|\Psi\rangle=\frac{1}{\sqrt{1+|\lambda|^{2}}}(|\phi\rangle+\lambda|\varphi\rangle) \quad(\lambda \in \mathbb{C}), \\
\text { with } \quad\left\{\begin{array}{l}
|\phi\rangle=\left|\phi_{1}\right\rangle \otimes\left|\phi_{2}\right\rangle, \quad|\varphi\rangle=\left|\varphi_{1}\right\rangle \otimes\left|\varphi_{2}\right\rangle, \\
\left\langle\phi_{1} \mid \phi_{1}\right\rangle=\left\langle\phi_{2} \mid \phi_{2}\right\rangle=\left\langle\varphi_{1} \mid \varphi_{1}\right\rangle=\left\langle\varphi_{2} \mid \varphi_{2}\right\rangle=1, \quad\left\langle\phi_{1} \mid \varphi_{1}\right\rangle=0
\end{array}\right.
\end{array}\right.
$$

so that $|\Psi\rangle$ is properly normalized.

For the moment, choose also

$$
\phi_{1}=\phi_{2} \equiv f \quad \text { and } \quad \varphi_{1}=\varphi_{2} \equiv g .
$$

with

$$
\langle f \mid f\rangle=\langle g \mid g\rangle=1, \quad\langle f \mid g\rangle=0 .
$$

Then

$$
\langle\Psi|\widehat{\mathcal{P}}| \Psi\rangle=\frac{1}{1+|\lambda|^{2}}\left[\langle\phi|\widehat{\mathcal{P}}| \phi\rangle+(\lambda\langle\phi|\widehat{\mathcal{P}}| \varphi\rangle+\text { c.c. })+|\lambda|^{2}\langle\varphi|\widehat{\mathcal{P}}| \varphi\rangle\right],
$$

with

$$
\begin{aligned}
\langle\phi|\widehat{\mathcal{P}}| \phi\rangle & =2\langle f|\widehat{\chi}| f\rangle+\left\langle f\left|\widehat{\chi}^{\prime}\right| f\right\rangle^{2}-\langle f|\widehat{\chi}| f\rangle^{2}-2\langle f|\widehat{\chi}| f\rangle\left\langle f\left|\widehat{\chi}^{\prime}\right| f\right\rangle \\
\langle\varphi|\widehat{\mathcal{P}}| \varphi\rangle & =2\langle g|\widehat{\chi}| g\rangle+\left\langle g\left|\widehat{\chi}^{\prime}\right| g\right\rangle^{2}-\langle g|\widehat{\chi}| g\rangle^{2}-2\langle g|\widehat{\chi}| g\rangle\left\langle g\left|\widehat{\chi}^{\prime}\right| g\right\rangle, \\
\langle\phi|\widehat{\mathcal{P}}| \varphi\rangle & =\left\langle f\left|\widehat{\chi}^{\prime}\right| g\right\rangle^{2}-\langle f|\widehat{\chi}| g\rangle^{2}-2\langle f|\widehat{\chi}| g\rangle\left\langle f\left|\widehat{\chi}^{\prime}\right| g\right\rangle .
\end{aligned}
$$


We already know that $0 \leq\langle\phi|\widehat{\mathcal{P}}| \phi\rangle \leq 1$ and $0 \leq\langle\varphi|\widehat{\mathcal{P}}| \varphi\rangle \leq 1$. Clearly, in view of (IV.17), our goal will be reached (namely $\langle\Psi|\widehat{\mathcal{P}}| \Psi\rangle<0$ or $\langle\Psi|\widehat{\mathcal{P}}| \Psi\rangle>1$ ) if one can find $f$ and $g$ such that

$$
|\langle\phi|\widehat{\mathcal{P}}| \varphi\rangle|^{2}>\langle\phi|\widehat{\mathcal{P}}| \phi\rangle\langle\varphi|\widehat{\mathcal{P}}| \varphi\rangle .
$$

We claim that this can be achieved with $S=S^{\prime}=(0, \infty), f(q) \equiv\langle q \mid f\rangle$ an even, normalized function in $L^{2}(-\infty, \infty)$, and

$$
g(q) \equiv\langle q \mid g\rangle=\operatorname{sgn}(q) f(q) .
$$

With this choice, eqs.(IV.16) are automatically satisfied and

$$
\langle f|\widehat{\chi}| f\rangle=\langle g|\widehat{\chi}| g\rangle=\langle f|\widehat{\chi}| g\rangle=\frac{1}{2}
$$

Also, since the Fourier transforms $\tilde{f}(p)$ and $\tilde{g}(p)$ are respectively even and odd functions

$$
\left\langle f\left|\widehat{\chi}^{\prime}\right| f\right\rangle=\left\langle g\left|\widehat{\chi}^{\prime}\right| g\right\rangle=\frac{1}{2} .
$$

As for the non trivial interference term $\left\langle f\left|\hat{\chi}^{\prime}\right| g\right\rangle$, it is given by (see appendix A)

$$
\left\langle f\left|\widehat{\chi}^{\prime}\right| g\right\rangle=-\frac{i}{\pi} \int_{0}^{\infty} d q \int_{0}^{\infty} d q^{\prime} f^{*}(q) f\left(q^{\prime}\right)\left(\frac{1}{q+q^{\prime}}-\frac{P}{q-q^{\prime}}\right) .
$$

At this stage, it is advantageous to take $f$ as a real function, so that by symmetry

$$
\left\langle f\left|\widehat{\chi}^{\prime}\right| g\right\rangle=-\frac{i}{\pi} \int_{0}^{\infty} d q \int_{0}^{\infty} d q^{\prime} \frac{f(q) f\left(q^{\prime}\right)}{q+q^{\prime}} .
$$

Let us set

$$
\begin{aligned}
h(q) & =\sqrt{2} f(q), \\
K\left(q, q^{\prime}\right) & =\frac{1}{\pi} \frac{1}{q+q^{\prime}} .
\end{aligned}
$$

Then

$$
\left\langle f\left|\widehat{\chi}^{\prime}\right| g\right\rangle=-\frac{i}{2} \gamma, \quad(\gamma \in \mathbb{R})
$$

with

$$
\gamma=\int_{0}^{\infty} d q \int_{0}^{\infty} d q^{\prime} h(q) K\left(q, q^{\prime}\right) h\left(q^{\prime}\right)
$$

and

$$
\|h\|_{L^{2}(0, \infty)}=1 \text {. }
$$

The insertion of eqs.(IV.21), (IV.22) and (IV.26) in (IV.18) gives

$$
\begin{gathered}
\langle\phi|\widehat{\mathcal{P}}| \phi\rangle=\langle\varphi|\widehat{\mathcal{P}}| \varphi\rangle=\frac{1}{2}, \\
\langle\phi|\widehat{\mathcal{P}}| \varphi\rangle=-\frac{1}{4}\left(1+\gamma^{2}\right)+\frac{i}{2} \gamma,
\end{gathered}
$$

so that eq.(IV.19) reads

$$
\left(\gamma^{2}+1\right)^{2}+4 \gamma^{2}>4
$$


which is satisfied provided that

$$
|\gamma|>\sqrt{2 \sqrt{3}-3} \cong 0.6813
$$

Moreover, with $\lambda=\rho \mathrm{e}^{i \theta}$, eq.(IV.17) becomes

$$
\langle\Psi|\widehat{\mathcal{P}}| \Psi\rangle=\frac{1}{2}-\frac{\rho}{2\left(1+\rho^{2}\right)}\left[\left(1+\gamma^{2}\right) \cos \theta+2 \gamma \sin \theta\right] .
$$

We already know that $|\gamma|$ cannot exceed 1, because $\left|\left\langle f\left|\widehat{\chi}^{\prime}\right| g\right\rangle\right|^{2} \leq\left\langle f\left|\widehat{\chi}^{\prime}\right| f\right\rangle\left\langle g\left|\widehat{\chi}^{\prime}\right| g\right\rangle=\frac{1}{4}$. Are there however some $h$ 's (subjected to (IV.28)) such that $\gamma$ (given by eq.([V.27)) fulfils (IV.29)? If this occurs we have reached our goal and it only remains to maximize $|\gamma|$ in order to obtain the extremal values of $\langle\Psi|\widehat{\mathcal{P}}| \Psi\rangle$ (within the present scheme) through eq.(IV.30). In other words, one has to solve the problem

$$
\gamma_{0} \equiv \sup _{\substack{\|h\|_{L^{2}(0, \infty)}=1 \\ h=h^{*}}}|\langle h|K| h\rangle|=?
$$

In appendix $\mathrm{B}$, it is shown that the (bounded) integral operator $K$ with kernel IV.25) on $L^{2}(0, \infty)$ is positive and has the purely continuous spectrum $[0,1]$. This immediately entails $\gamma_{0}=1$, and we get

$$
\begin{gathered}
\left.\langle\Psi|\widehat{\mathcal{P}}| \Psi\rangle\right|_{\gamma=1}=\frac{1}{2}-\frac{\rho}{1+\rho^{2}}(\cos \theta+\sin \theta) \\
\left.\inf _{\lambda}\langle\Psi|\widehat{\mathcal{P}}| \Psi\rangle\right|_{\gamma=1}=\left.\langle\Psi|\widehat{\mathcal{P}}| \Psi\rangle\right|_{\gamma=\rho=1, \theta=\frac{\pi}{4}}=\frac{1-\sqrt{2}}{2} \cong-0.2071 \\
\left.\sup _{\lambda}\langle\Psi|\widehat{\mathcal{P}}| \Psi\rangle\right|_{\gamma=1}=\left.\langle\Psi|\widehat{\mathcal{P}}| \Psi\rangle\right|_{\gamma=\rho=1, \theta=\frac{-3 \pi}{4}}=\frac{1+\sqrt{2}}{2} \cong 1.2071
\end{gathered}
$$

Actually, as discussed in appendix B, due to the continuous spectrum of $K$, these extremal values cannot be strictly reached, but only approached arbitrarily close via a family of normalized functions $h$, e.g.

$$
h_{L}(q)=\frac{\theta(L-q)}{\sqrt{\ln (L+1)}} \frac{1}{\sqrt{q+1}}, \quad L \rightarrow \infty
$$

or smoothed forms of this. Of course, other functions $h$ will also do the job (although less perfectly), that is meet the crucial requirement (IV.29). Taking for example $h(q)=\frac{1}{q+1}\left(\right.$ which is normalized in $\left.L^{2}(0, \infty)\right)$, one gets

$$
\gamma=\frac{\pi}{4} \cong 0.7854
$$

Finally, collecting the equations (IV.14), (IV.15), (IV.20) and (IV.24), together with $\lambda=\frac{\pi}{4},-\frac{3 \pi}{4}$, one obtains the wave functions leading to the maximal violations (IV.31)

$$
\Psi_{ \pm}\left(q_{1}, q_{2}\right)=\frac{1}{2 \sqrt{2}}\left[1 \pm \mathrm{e}^{i \frac{\pi}{4}} \operatorname{sgn}\left(q_{1}\right) \operatorname{sgn}\left(q_{2}\right)\right] h\left(\left|q_{1}\right|\right) h\left(\left|q_{2}\right|\right)
$$

where $h(q)$ stands for some regularized form of $\frac{1}{\sqrt{q}}$, with $\int_{0}^{\infty} d q h(q)^{2}=1$. 


\section{IV-C. Introducing Einstein locality and relative motion}

Let us now interpret $q_{1}$ and $q_{2}$ as the coordinates of two particles (rather than the $x$ and $y$ coordinates of the same particle).. Then the wave functions (IV.32) describe states of two particles not spatially separated and with zero relative momentum. These two restrictions can be easily disposed of.

First, it can be checked that nothing is essentially changed in the previous derivation if one keeps

$$
S_{1}=S_{1}^{\prime}=(0, \infty) ; \quad \phi_{1}\left(q_{1}\right)=f\left(q_{1}\right), \quad \varphi_{1}\left(q_{1}\right)=\operatorname{sgn}\left(q_{1}\right) f\left(q_{1}\right),
$$

but replaces

$$
S_{2}=S_{2}^{\prime}=(0, \infty) ; \quad \phi_{2}\left(q_{2}\right)=f\left(q_{2}\right), \quad \varphi_{2}\left(q_{2}\right)=\operatorname{sgn}\left(q_{2}\right) f\left(q_{2}\right),
$$

by

$S_{2}=(a, \infty), \quad S_{2}^{\prime}=(0, \infty) ; \quad \phi_{2}\left(q_{2}\right)=f\left(q_{2}-a\right), \quad \varphi_{2}\left(q_{2}\right)=\operatorname{sgn}\left(q_{2}-a\right) f\left(q_{2}-a\right)$.

Then eq. (IV.32) becomes

$$
\Psi_{ \pm}\left(q_{1}, q_{2}\right)=\frac{1}{2 \sqrt{2}}\left[1 \pm \mathrm{e}^{i \frac{\pi}{4}} \operatorname{sgn}\left(q_{1}\right) \operatorname{sgn}\left(q_{2}-a\right)\right] h\left(\left|q_{1}\right|\right) h\left(\left|q_{2}-a\right|\right)
$$

with $a$ arbitrary.

This allows us to let Einstein locality enter the game.

Similarly, nothing is essentially changed if one keeps eqs.(IV.33) but replaces eqs. (IV.34) by

$$
S_{2}=(0, \infty), \quad S_{2}^{\prime}=(P, \infty) ; \quad \phi_{2}\left(q_{2}\right)=\mathrm{e}^{i P q_{2}} f\left(q_{2}\right), \quad \varphi_{2}\left(q_{2}\right)=\mathrm{e}^{i P q_{2}} \operatorname{sgn}\left(q_{2}\right) f\left(q_{2}\right) .
$$

Then eq.(IV.32) becomes

$$
\Psi_{ \pm}\left(q_{1}, q_{2}\right)=\frac{1}{2 \sqrt{2}}\left[1 \pm \mathrm{e}^{i \frac{\pi}{4}} \operatorname{sgn}\left(q_{1}\right) \operatorname{sgn}\left(q_{2}\right)\right] \mathrm{e}^{i P q_{2}} h\left(\left|q_{1}\right|\right) h\left(\left|q_{2}\right|\right),
$$

with $P$ arbitrary.

This allows us to put the two particles in relative motion.

\section{IV-D. Analogy with Bell spin $\frac{1}{2}$ correlation inequalities}

Let us denote by $|+\rangle$ a normalized function $f$ close to the (symmetrized) eigenfunction of the operator $K$ with "eigenvalue" $\lambda_{0}=1$ (i.e. $\gamma \cong 1$ in eq.([V.26)), and by $|-\rangle$ the orthogonal function $g$ (as given by eq.(IV.20)). Consider the subspace $V=\operatorname{span}(|+\rangle,|-\rangle)$ of the full 1-particle Hilbert space, together with the orthogonal projector $\Pi$ onto $V$. Call $\Gamma$ (resp. $\Gamma^{\prime}$ ) the restriction of $\hat{\chi}$ (resp. $\hat{\chi}^{\prime}$ ) to the 2-dimensional space $V$

$$
\Gamma=\Pi \widehat{\chi} \Pi, \quad \Gamma^{\prime}=\Pi \widehat{\chi}^{\prime} \Pi .
$$


Then eqs.(IV.21), (IV.22) and (IV.26) tell us that $\Gamma$ and $\Gamma^{\prime}$ are represented in the orthonormal basis $\{|+\rangle,|-\rangle\}$ by the matrices

$$
\begin{gathered}
\Gamma=\left(\begin{array}{cc}
\frac{1}{2} & \frac{1}{2} \\
\frac{1}{2} & \frac{1}{2}
\end{array}\right)=\frac{1}{2}\left(1+\sigma_{x}\right), \\
\Gamma^{\prime}=\left(\begin{array}{cc}
\frac{1}{2} & \frac{i}{2} \gamma \\
-\frac{i}{2} \gamma & \frac{1}{2}
\end{array}\right)=\frac{1}{2}\left(1-\gamma \sigma_{y}\right) .
\end{gathered}
$$

In the idealized limit $\gamma \rightarrow 1$ (and only in this limit), one observes that $\Gamma$ and $\Gamma^{\prime}$ are themselves orthogonal projections $V \rightarrow V$

$$
\begin{aligned}
\Gamma & =\Gamma^{\dagger}, & \Gamma^{2} & =\Gamma, \\
\Gamma^{\prime} & =\Gamma^{\prime \dagger}, & \Gamma^{\prime 2} & =\Gamma^{\prime} .
\end{aligned}
$$

This implies that both operators $\widehat{\chi}$ and $\widehat{\chi}^{\prime}$ leave the subspace $V$ invariant

$$
[\Pi, \widehat{\chi}]=\left[\Pi, \widehat{\chi}^{\prime}\right]=0 .
$$

Indeed, a straightforward calculation shows that

$$
[(\mathbf{1}-\Pi) \widehat{\chi} \Pi]^{\dagger}[(\mathbf{1}-\Pi) \widehat{\chi} \Pi]=0,
$$

which entails $(\mathbf{1}-\Pi) \widehat{\chi} \Pi=0$ and $\widehat{\chi} \Pi=\Pi \widehat{\chi}$. The same for $\widehat{\chi}^{\prime}$.

Hence, in the 2-particle Hilbert space, the operator (IV.13) also leaves invariant $V \otimes V$, and $\overline{\mathcal{P}}:=\widehat{\mathcal{P}} \Pi$ assumes the simple form

$$
\overline{\mathcal{P}}=\frac{1}{2}+\frac{1}{4}\left(\sigma_{y}^{(1)} \sigma_{y}^{(2)}-\sigma_{x}^{(1)} \sigma_{x}^{(2)}+\sigma_{x}^{(1)} \sigma_{y}^{(2)}+\sigma_{y}^{(1)} \sigma_{x}^{(2)}\right),
$$

whereas the maximally violating wave functions (IV.32) read

$$
\left|\Psi_{ \pm}\right\rangle=\frac{1}{\sqrt{2}}\left(|+\rangle^{(1)}|+\rangle^{(2)} \pm \mathrm{e}^{i \frac{\pi}{4}}|-\rangle^{(1)}|-\rangle^{(2)}\right) .
$$

From eq.(IV.35) one can check that

$$
\overline{\mathcal{P}}(\mathbf{1}-\overline{\mathcal{P}})=-\frac{1}{4} \sigma_{z}^{(1)} \sigma_{z}^{(2)},
$$

which is just the projected form of $\widehat{\mathcal{P}}(\mathbf{1}-\widehat{\mathcal{P}})=\left[\widehat{\chi}_{1}, \widehat{\chi}_{1}^{\prime}\right]\left[\widehat{\chi}_{2}, \widehat{\chi}_{2}^{\prime}\right]$, and the expectation value of the operator (IV.37) is

$$
\left\langle\Psi_{ \pm}|\overline{\mathcal{P}}(\mathbf{1}-\overline{\mathcal{P}})| \Psi_{ \pm}\right\rangle=-\frac{1}{4}
$$

for the wave functions (IV.36).

The result (IV.31) is also directly recovered from eqs.(IV.35) and (IV.36)

$$
\left\langle\Psi_{ \pm}|\overline{\mathcal{P}}| \Psi_{ \pm}\right\rangle=\frac{1 \mp \sqrt{2}}{2}
$$

Then one sees that, in the idealized limit $\gamma \rightarrow 1$, the original phase space setting up of the problem is formally equivalent to the standard EPR setting up for a two spin $\frac{1}{2}$ system, together with its classical Bell inequalities. 


\section{General solution of the three marginal problem}

We have proved here the impossibility of reproducing quantum probabilities of four CCS as marginals. Roy and Singh [7] have given examples to show that reproducing three CCS is possible. In this section, we construct the most general nonnegative phase space density which reproduces three different (noncommuting) CCS as marginals. Our results encapsulate the extent to which noncommuting CCS can be simultaneously realized in quantum mechanics.

Among the four marginals $R, S, T, U$ obeying the compatibility conditions (II.7) which are at our disposal, the particular choice of three of them is completely irrelevant. For definiteness, we choose $R, T$ and $U$, which we rename $\sigma_{0}\left(q_{1}, q_{2}\right), \sigma_{1}\left(p_{1}, q_{2}\right)$ and $\sigma_{2}\left(p_{1}, p_{2}\right)$.

We assume that these marginals are probability densities in the full mathematical sense, that is they are true (integrable and non negative) functions. This means that we restrict our marginal probability distributions to absolutely continuous measures (with respect to Lebesgue measure) in $\mathbb{R}^{2}$. Notice that such a restriction is automatic in the quantum case, due to eqs.(II.8).

Likewise, we look for the general solution of the three marginal problem in the class of absolutely continuous measures in the phase space $\mathbb{R}^{4}$. This means that we want to describe all the solutions $\rho$ of the equations

$$
\begin{aligned}
& \sigma_{0}\left(q_{1}, q_{2}\right)=\int d p_{1} d p_{2} \rho(\vec{q}, \vec{p}), \\
& \sigma_{1}\left(p_{1}, q_{2}\right)=\int d q_{1} d p_{2} \rho(\vec{q}, \vec{p}), \\
& \sigma_{2}\left(p_{1}, p_{2}\right)=\int d q_{1} d q_{2} \rho(\vec{q}, \vec{p}),
\end{aligned}
$$

which belong to $L^{1}\left(\mathbb{R}^{4}, d^{2} q d^{2} p\right)$.

Notice that this is a restricted problem even in the quantum case, since nothing prevents a probability measure containing a singular part to project on marginals which are $L^{1}$-functions. To some extent, the above restrictions can be removed, allowing us to include e.g. probability measures partly concentrated on submanifolds of the phase space. However, dealing with such extensions at some degree of generality requires painful manipulations, and we shall ignore them heref As for the full inclusion of singular measures, it appears as both delicate and of little practical interest.

Let us introduce the one variable marginals

$$
\begin{aligned}
& \sigma_{01}\left(q_{2}\right)=\int d q_{1} \sigma_{0}\left(q_{1}, q_{2}\right), \\
& \sigma_{12}\left(p_{1}\right)=\int d q_{2} \sigma_{1}\left(p_{1}, q_{2}\right) .
\end{aligned}
$$

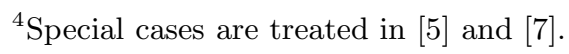


Owing to the compatibility conditions (II.7), these definitions are equivalent to

$$
\begin{aligned}
\sigma_{01}\left(q_{2}\right) & =\int d p_{1} \sigma_{1}\left(p_{1}, q_{2}\right), \\
\sigma_{12}\left(p_{1}\right) & =\int d p_{2} \sigma_{2}\left(p_{1}, p_{2}\right) .
\end{aligned}
$$

As the support properties of the functions $\sigma_{j}$ (which are allowed to vanish on some parts of $\mathbb{R}^{2}$ ) are not innocent in the forthcoming construction, we need to pay attention to them. Let $\Sigma_{j} \subset \mathbb{R}^{2}(j=0,1,2)$ be the essential support of $\sigma_{j}$. The above compatibility conditions, together with the positivity conditions $\sigma_{j} \geq 0$, clearly yield two constraints on the supports $\Sigma_{j}$, namely

$$
\begin{aligned}
& \left\{q_{2} \in \mathbb{R} \mid \exists q_{1} \in \mathbb{R} \text { such that }\left(q_{1}, q_{2}\right) \in \Sigma_{0}\right\} \quad= \\
& \left\{q_{2} \in \mathbb{R} \mid \exists p_{1} \in \mathbb{R} \text { such that }\left(p_{1}, q_{2}\right) \in \Sigma_{1}\right\}, \\
& \left\{p_{1} \in \mathbb{R} \mid \exists q_{2} \in \mathbb{R} \text { such that }\left(p_{1}, q_{2}\right) \in \Sigma_{1}\right\}= \\
& \left\{p_{1} \in \mathbb{R} \mid \exists p_{2} \in \mathbb{R} \text { such that }\left(p_{1}, p_{2}\right) \in \Sigma_{2}\right\} \text {. }
\end{aligned}
$$

To the $\Sigma_{j}$ 's we associate the subsets $E_{j}$ 's of the phase space defined by

$$
\begin{aligned}
& E_{0}=\left\{\vec{q}, \vec{p} \mid\left(q_{1}, q_{2}\right) \in \Sigma_{0},\left(p_{1}, p_{2}\right) \in \mathbb{R}^{2}\right\}, \\
& E_{1}=\left\{\vec{q}, \vec{p} \mid\left(p_{1}, q_{2}\right) \in \Sigma_{1},\left(q_{1}, p_{2}\right) \in \mathbb{R}^{2}\right\}, \\
& E_{2}=\left\{\vec{q}, \vec{p} \mid\left(p_{1}, p_{2}\right) \in \Sigma_{2},\left(q_{1}, q_{2}\right) \in \mathbb{R}^{2}\right\} .
\end{aligned}
$$

Finally, we denote by $E$ the intersection of the $E_{j}$ 's

$$
E=E_{0} \cap E_{1} \cap E_{2}
$$

Clearly, due to positivity again, any solution $\rho$ of eqs. (V.1) must have its essential support contained in $E$.

The three marginal problem in the precise form stated above is then completely solved by

Theorem 1 1) The Lebesgue measure of $E$ is not zero and the function $\rho_{0}$ defined (a.e.) by

$$
\rho_{0}(\vec{q}, \vec{p})=\left\{\begin{array}{l}
\sigma_{0}\left(q_{1}, q_{2}\right) \frac{1}{\sigma_{01}\left(q_{2}\right)} \sigma_{1}\left(p_{1}, q_{2}\right) \frac{1}{\sigma_{12}\left(p_{1}\right)} \sigma_{2}\left(p_{1}, p_{2}\right) \quad \text { if }(\vec{q}, \vec{p}) \in E, \\
0 \quad \text { otherwise, }
\end{array}\right.
$$

is a non negative solution of the problem (V.1) in $L^{1}\left(\mathbb{R}^{4}, d^{2} q d^{2} p\right)$.

2) The general solution $\rho$ of (V.1) in $L^{1}\left(\mathbb{R}^{4}, d^{2} q d^{2} p\right)$ is given by

$$
\rho(\vec{q}, \vec{p})=\rho_{0}(\vec{q}, \vec{p})+\lambda \Delta(\vec{q}, \vec{p}),
$$

where

$$
\lambda \in\left[-\frac{1}{m_{+}}, \frac{1}{m_{-}}\right]
$$


and

$$
\begin{aligned}
& \Delta(\vec{q}, \vec{p})=F(\vec{q}, \vec{p})-\rho_{0}(\vec{q}, \vec{p})\left[\frac{1}{\sigma_{0}\left(q_{1}, q_{2}\right)} \int d p_{1}^{\prime} d p_{2}^{\prime} F\left(q_{1}, q_{2}, p_{1}^{\prime}, p_{2}^{\prime}\right)\right. \\
& \quad+\frac{1}{\sigma_{1}\left(p_{1}, q_{2}\right)} \int d q_{1}^{\prime} d p_{2}^{\prime} F\left(q_{1}^{\prime}, q_{2}, p_{1}, p_{2}^{\prime}\right)+\frac{1}{\sigma_{2}\left(p_{1}, p_{2}\right)} \int d q_{1}^{\prime} d q_{2}^{\prime} F\left(q_{1}^{\prime}, q_{2}^{\prime}, p_{1}, p_{2}\right) \\
& \left.\quad-\frac{1}{\sigma_{01}\left(q_{2}\right)} \int d q_{1}^{\prime} d p_{1}^{\prime} d p_{2}^{\prime} F\left(q_{1}^{\prime}, q_{2}, p_{1}^{\prime}, p_{2}^{\prime}\right)-\frac{1}{\sigma_{12}\left(p_{1}\right)} \int d q_{1}^{\prime} d q_{2}^{\prime} d p_{2}^{\prime} F\left(q_{1}^{\prime}, q_{2}^{\prime}, p_{1}, p_{2}^{\prime}\right)\right],
\end{aligned}
$$

$F$ being an arbitrary $L^{1}\left(\mathbb{R}^{4}, d^{2} q d^{2} p\right)$-function with essential support contained in $E$. The (F-dependent) constants $m_{ \pm}$in (V.9) are defined as

$$
m_{+}=\underset{(\vec{q}, \vec{p}) \in E}{\operatorname{ess} \sup _{0}} \frac{\Delta(\vec{q}, \vec{p})}{\rho_{0}(\vec{q}, \vec{p})}, \quad m_{-}=-\underset{(\vec{q}, \vec{p}) \in E}{\operatorname{essinf}} \frac{\Delta(\vec{q}, \vec{p})}{\rho_{0}(\vec{q}, \vec{p})},
$$

and are both positive if $\Delta \neq 0\left(m_{+}=\infty\right.$ or/and $m_{-}=\infty$ are not excluded).

Proof:

1) To begin with, $\rho_{0}$ given by (V.7) is well defined and non negative. Indeed, due to (V.2) (or (V.3)) and the positivity of the $\sigma_{j}$ 's, $\sigma_{01}\left(q_{2}\right)$ and $\sigma_{12}\left(p_{1}\right)$ are a.e. non zero for $(\vec{q}, \vec{p}) \in E_{0}$ and $E_{1}$ (or $E_{1}$ and $E_{2}$ ), so that the denominators in eq. (V.7) do not vanish on $E$ (except maybe on sets of Lebesgue measure 0 ).

Next, in order to check that $\rho_{0}$ obeys the first equation (V.1), we consider the integral

$$
\int d p_{1} \int d p_{2} \rho_{0}(\vec{q}, \vec{p})
$$

with this specific order of the $p$ integrations. According to the relations (V.4) and the definition of $E$, one observes first that the projection of $E$ on the $\left(p_{1}, p_{2}\right)$ plane is the set $\Sigma_{2}$, so that the integration over $p_{2}$ removes the factor $\sigma_{2} / \sigma_{12}$ in $\rho_{0}$; and second, that the projections of $\Sigma_{1}$ and $\Sigma_{2}$ on $p_{1}$ coincide, so that the integration over $p_{1}$ removes the factor $\sigma_{1} / \sigma_{01}$ in $\rho_{0}$, and one is left with the expected result $\sigma_{0}\left(q_{1}, q_{2}\right)$. We can now write

$$
\int d p_{1} d p_{2} \rho_{0}(\vec{q}, \vec{p})=\sigma_{0}\left(q_{1}, q_{2}\right)
$$

where, thanks to Fubini theorem, the integration order is completely irrelevant. The other two equations (V.1) are derived in a similar way.

This calculation shows at once that the Lebesgue measure of $E$ is not zero and that $\rho_{0} \in L^{1}\left(\mathbb{R}^{4}, d^{2} q d^{2} p\right)$.

2) That any non negative solution $\rho$ of eqs.(V.1) admits the representation (V.8)(V.10) is easy to establish. Indeed, since the essential support of $\rho$ is necessarily contained in $E$, we are allowed to take $F=\rho$ in eq.(V.10), which gives (using (V.1))

$$
\Delta(\vec{q}, \vec{p})=\rho(\vec{q}, \vec{p})-\rho_{0}(\vec{q}, \vec{p}) .
$$

Then, from (V.11)

$$
m_{-}=-\underset{(\vec{q}, \vec{p}) \in E}{\operatorname{ess} \inf _{0}}\left(\frac{\rho}{\rho_{0}}-1\right) \leq 1
$$


As $1 / m_{-} \geq 1$ in (V.9), we can choose $\lambda=1$, which makes eq. (V.14) equivalent to the representation (V.8).

It remains to show that any function $\rho$ defined by $(\mathrm{V} .8)$ to $(\mathrm{V} .11)$ (and thus with essential support $E$ ) is a non negative solution of eqs. (V.1) in $L^{1}\left(\mathbb{R}^{4}, d^{2} q d^{2} p\right)$. In order to prove that $\rho$ satisfies the first equation (V.1), we rearrange pairwise the right-hand side of $(\overline{V .10})$ as follows

$$
\begin{aligned}
\Delta= & {\left[F-\frac{\rho_{0}}{\sigma_{0}} \int d p_{1}^{\prime} d p_{2}^{\prime} F\right]-\left[\frac{\rho_{0}}{\sigma_{1}} \int d q_{1}^{\prime} d p_{2}^{\prime} F-\frac{\rho_{0}}{\sigma_{01}} \int d q_{1}^{\prime} d p_{1}^{\prime} d p_{2}^{\prime} F\right] } \\
& -\left[\frac{\rho_{0}}{\sigma_{2}} \int d q_{1}^{\prime} d q_{2}^{\prime} F-\frac{\rho_{0}}{\sigma_{12}} \int d q_{1}^{\prime} d q_{2}^{\prime} d p_{2}^{\prime} F\right] .
\end{aligned}
$$

Then, integrating the right-hand side over $p_{1}$ and $p_{2}$, one finds, by an extensive use of eqs. (V.2) to (V.4) as in part 1), that the two terms coming from each square bracket cancel each other, leading to

$$
\int d p_{1} d p_{2} \Delta(\vec{q}, \vec{p})=0
$$

This, with (V.8) and (V.13), implies that $\rho$ satisfies the first equation (V.1). That it satisfies the other two equations (V.1) is proved in a similar way.

This calculation also shows that $\rho \in L^{1}\left(\mathbb{R}^{4}, d^{2} q d^{2} p\right)$.

Finally

$$
\int_{E} d^{2} q d^{2} p \Delta(\vec{q}, \vec{p})=0,
$$

which implies that $m_{ \pm}$in eqs. (V.11) are both strictly positive if $\Delta$ does not vanish a.e. on $\mathbb{R}^{4}$. The positivity of $\rho$ is then a trivial consequence of eqs. $(\overline{\mathrm{V} .8})$, $(\overline{\mathrm{V} .9})$ and (V.11).

The proof is complete.

Remark:

Theorem 1, as it is stated above, deals with $L^{1}$ functions, and thus excludes the occurrence of Dirac measures. We insist on the fact that this is unnecessarily restrictive. Indeed Dirac measures can be easily accommodated and the theorem suitably rephrased, to the price however of cumbersome mathematical intricacies which we do not want to enter into.

An immediate corollary of Proposition 1 and Theorem 1 is

Theorem 2 (Three marginal theorem) Let $R, S, T$ and $U$ be probablilty distributions for $\left(q_{1}, q_{2}\right),\left(q_{1}, p_{2}\right),\left(p_{1}, q_{2}\right)$ and $\left(p_{1}, p_{2}\right)$ obeying the consistency conditions (II. X). Given $n$ arbitrary distributions among $\{R, S, T, U\}$, a necessary and sufficient condition for them to be marginals of a probability density in the 4-dimensional phase space is $n \leq 3$.

\section{Conclusions}

We have solved the four marginal problem in four dimensional phase space thus proving a long standing conjecture [7] and vastly improving the first results of Martin 
and Roy [1] which dealt with infinite number of marginals. To achieve this, we first derived "phase space Bell inequalities" which have their own interest. Actually they allow, at least in principle, direct "experimental" tests of the orthodox-versus-hidden variable interpretations of quantum mechanics within the position-momentum sector, analoguous to those performed within the spin sector.

The technique of phase space Bell inequalities established here has applications to quantum information processing. Generalizing the example (IV.11), one can show that for any separable density operator $\rho$ one can construct a phase space density obeying the four marginal conditions. Hence, the Bell inequalities ([II.2), with $R, S$, $T$ and $U$ given by (II.8) must hold for every separable quantum state, irrespective of any physical interpretation of the associated phase space density. Their violation by a quantum state is a signature and even a quantitative measure of entanglement of this state.

We have also constructed the most general positive definite phase space density which has the maximum number of marginals (three) coinciding with corresponding quantum probabilities of three different (noncommuting) CCS. These results should be useful in the construction of maximally realistic quantum theories.

\section{Acknowledgements}

We thank André Martin for collaboration in the initial stages of this work. One of us (SMR) thanks A. Fine and A. Garg for some remarks on the three marginal problem many years ago. 


\section{Appendix A. Proof of equation (ㅍ.23)}

Since $S^{\prime}=(0, \infty)$, one has

$$
\widehat{\chi}^{\prime}(p) \tilde{g}(p)=\theta(p) \tilde{g}(p) .
$$

Assuming first that $g$ belongs to $\mathcal{S}$ (the Schwartz space of infinitely differentiable functions on $\mathbb{R}$ with fast decrease at infinity), one can write

$$
\left(\widehat{\chi}^{\prime} g\right)(q)=\int_{-\infty}^{\infty} d q^{\prime} \tilde{\theta}\left(q-q^{\prime}\right) g\left(q^{\prime}\right)
$$

where $\tilde{\theta}$ is the Fourier transform of $\theta$ in the distribution-theoretic sense

$$
\tilde{\theta}(q) \equiv \frac{1}{2 \pi} \int_{-\infty}^{\infty} d p \mathrm{e}^{i p q} \theta(p)=\frac{i}{2 \pi} \frac{P}{q}+\frac{1}{2} \delta(q) .
$$

Then, if $f$ also belongs to $\mathcal{S}$

$$
\left\langle f\left|\widehat{\chi}^{\prime}\right| g\right\rangle=\frac{i}{2 \pi} \int_{-\infty}^{\infty} d q f^{*}(q) \int_{-\infty}^{\infty} d q^{\prime} \frac{P}{q-q^{\prime}} g\left(q^{\prime}\right)+\frac{1}{2}\langle f \mid g\rangle .
$$

In particular, for even $f$ and odd $g,\langle f \mid g\rangle$ vanishes, and

$$
\left\langle f\left|\hat{\chi}^{\prime}\right| g\right\rangle=-\frac{i}{\pi} \int_{0}^{\infty} d q f^{*}(q) \int_{0}^{\infty} d q^{\prime}\left(\frac{1}{q+q^{\prime}}-\frac{P}{q-q^{\prime}}\right) g\left(q^{\prime}\right),
$$

which gives eq. (IV.23) if $g$ coincides with $f$ on $(0, \infty)$. The continuation from $\mathcal{S}$ to $L^{2}(-\infty, \infty)$ is performed as usual by continuity, using the fact that $\mathcal{S}$ is a dense subspace in $L^{2}(-\infty, \infty)$.

\section{Appendix B. Study of the operator K}

From the very definition of $K$ through the integral kernel (IV.25), one has

$$
(K h)(q)=\frac{1}{\pi} \int_{0}^{\infty} d q^{\prime} \frac{h\left(q^{\prime}\right)}{q+q^{\prime}} .
$$

Let us put

$$
\bar{h}(u)=\mathrm{e}^{\frac{u}{2}} h\left(\mathrm{e}^{u}\right) .
$$

Since $\int_{-\infty}^{\infty} d u|\bar{h}(u)|^{2}=\int_{0}^{\infty} d q|h(q)|^{2}$, the correspondence $h \mapsto \bar{h}$ defines a unitary mapping $L^{2}(0, \infty) \rightarrow L^{2}(-\infty, \infty)$ and

$$
\overline{K h}(u)=\int_{-\infty}^{\infty} d v \bar{K}(u-v) \bar{h}(v)
$$

where

$$
\bar{K}(u)=\frac{1}{2 \pi \cosh \frac{u}{2}} .
$$

Then, another unitary mapping $L^{2}(-\infty, \infty) \rightarrow L^{2}(-\infty, \infty)$, namely the Fourier transform

$$
\widetilde{\bar{h}}(k)=\frac{1}{\sqrt{2 \pi}} \int_{-\infty}^{\infty} d u \mathrm{e}^{i k u} \bar{h}(u)
$$


reduces the convolution product in (B.1) to an ordinary product

$$
\widetilde{\overline{K h}}(k)=\widetilde{\bar{K}}(k) \widetilde{\bar{h}}(k) \text {, }
$$

where

$$
\widetilde{\bar{K}}(k) \equiv \int_{-\infty}^{\infty} d u \mathrm{e}^{i k u} \bar{K}(u)=\frac{1}{\cosh \pi k} .
$$

Therefore, the operator $K$ on $L^{2}(0, \infty)$ is unitarily equivalent to the multiplicative operator $(B .2)$ on $L^{2}(-\infty, \infty)$. The latter is evidently a positive operator with purely continuous spectrum $[0,1]$. Its generalized (non normalizable) "eigenfunctions" are

$$
\widetilde{\bar{h}}_{s}(k)=\delta(k-s) \quad(s \in \mathbb{R}),
$$

with "eigenvalues" $\lambda_{s}=\frac{1}{\cosh \pi s}$, and their preimage in $L^{2}(0, \infty)$ are

$$
h_{s}(q)=\frac{1}{\sqrt{2 \pi q}} \mathrm{e}^{-i s \ln q} .
$$

Of particular interest for us is the extremal one, with "eigenvalue" $\lambda_{0}=1$

$$
h_{0}(q)=\frac{1}{\sqrt{2 \pi q}} .
$$

Of course, the corresponding maximal value $\gamma_{0}=1$ of $\gamma=\langle h|K| h\rangle$ cannot be attained, but only approached arbitrarily close through a family of normalizable functions mimicking $\frac{1}{\sqrt{q}}$. For instance, introducing two cutoffs, $\varepsilon$ at small $q$ and $L$ at large $q$, and setting

$$
h_{\varepsilon, L}(q)=\frac{1}{\sqrt{\ln \frac{L}{\varepsilon}}} \chi_{(\varepsilon, L)}(q) \frac{1}{\sqrt{q}} \quad\left(\left\|h_{\varepsilon, L}\right\|=1\right)
$$

one gets

$$
\left\langle h_{\varepsilon, L}|K| h_{\varepsilon, L}\right\rangle=1-\frac{4}{\pi \ln \frac{L}{\varepsilon}} \int_{\sqrt{\varepsilon / L}}^{1} d x \frac{\arctan x}{x}=1-\mathrm{O}\left(\frac{1}{\ln \frac{L}{\varepsilon}}\right),
$$

so that $\lim _{\substack{\varepsilon \rightarrow 0 \\ L \rightarrow \infty}}\left\langle h_{\varepsilon, L}|K| h_{\varepsilon, L}\right\rangle=1$.

Notice that one can keep $\varepsilon$ fixed (e.g. $\varepsilon=1$ ) and let $L$ alone go to $\infty$ without changing anything (this is in fact a consequence of the scale invariance of the operator $K$ ), or even choose a family of less singular functions $h$, like

$$
h_{L}(q)=\frac{1}{\sqrt{\ln (L+1)}} \theta(L-q) \frac{1}{\sqrt{q+1}}
$$

\section{References}

[1] A. Martin and S.M. Roy, Phys. Lett. B350, 66 (1995).

[2] A.M. Gleason, J. Math. Mech. 6, 885 (1957); S. Kochen and E.P. Specker, J. Math. Mech. 17, 59 (1967); J.S. Bell, Rev. Mod. Phys. 모, 447 (1966). 
[3] E. Wigner, Phys. Rev. 뜨, 749 (1932).

[4] L. Cohen, Y.I. Zaparovanny, J. Math. Phys. 21, 794 (1980); L. Cohen, J. Math. Phys. 25, 2402 (1984).

[5] S.M. Roy, V. Singh, Mod. Phys. Lett. $\underline{\text { A10, }} 709$ (1995).

[6] L. de Broglie, "Nonlinear Wave Mechanics, A Causal Interpretation", Elsevier, 1960; D. Bohm, Phys. Rev. $\underline{85}, 166$ (1952) and ㅌ55, 180 (1952); P.R. Holland, "The Quantum Theory of Motion", Cambridge Univ. Press, 1993; D. Bohm, B.J. Hiley, "The Undivided Universe", Routledge, London, 1993.

[7] S.M. Roy and V. Singh, Phys. Letters A255, 201 (1999).

[8] J.S. Bell, Physics 1, 195 (1964).

[9] L. Cohen, Proc. ICASSP 모, 41B1.1 (1984); L. Cohen, "Time-Frequency Analysis", Prentice Hall, Englewood Cliffs, N.J. (1995).

[10] G. Auberson, G. Mahoux, S.M. Roy, V. Singh, "Bell inequalities in (2N)dimensional phase space and the $(\mathrm{N}+1)$ marginal theorem", in preparation.

[11] G. Auberson, G. Mahoux, S.M. Roy, V. Singh, "Bell inequalities in phase space and their violation in quantum mechanics", quant-ph/0205157, preprint TIFR/TH/02-18 (Mumbai, India) and PM/02-14 (Montpellier, France). 